\title{
Combating resistance: infectious diseases
}

\author{
"Some of the most deadly infectious diseases affect the poorest areas of the world and resistance to what few regimens are \\ available in a poorly funded healthcare system can lead to devastating effects."
}

The development of resistance to drugs used in the treatment of infectious diseases and cancers is becoming an increasing global health burden. Both therapeutic areas have had large quantities of money and man hours invested into tackling these problems in recent years, attracting attention and research from academia, industry, charities and governmental institutions with financial support being provided through both public and private organizations.

Cancer and infectious diseases differ in that resistance to treatments for the latter leads to the spread of infectious diseases. Some of the most deadly infectious diseases affect the poorest areas of the world and resistance to what few regimens are available in a poorly funded healthcare system can lead to devastating effects.

Resistance to anti-infective agents has received a lot of public press in recent years with so-called hospital super bugs and concerns regarding resistant strains of common infection-causing bacteria. Outbreaks of influenza viruses have also led to public discussion relating to whether suitable antivirals are available and whether they are effective enough to treat the magnitude of the situation.

Following on from volume 5, issue 9 [1-9], which was devoted to combating cancer resistance, the second part of this special edition of Future Medicinal Chemistry features articles relating to infectious disease drug resistance covering diseases, such as malaria and influenza HIV.

The issue starts with a trio of opinion-based Editorials. John Manchester provides an industrial perspective on antibacterial drug discovery and updates us on the activity of the Innovative Medicines Initiative [10]. Amy Anderson analyzes how close we are to modeling drug resistance and how this can accelerate drug discovery [11] and Latour and Durate question whether PerR can be targeted in the future [12]. In an Interview with Future Medicinal Chemistry, Celia Schiffer updates us on her research and discussed the setting up the Institute for Drug Resistance and how those involved in the field should consider evolution when designing new drugs. Speaking to the journal, Line Matthiessen (European Commission), discusses the Unit of Infectious Diseases and Public Health's current work with regard to combating resistance to antimicrobial drugs, including those used to treat neglected diseases [13,14].

There are two Perspective articles featured, one from Chaoping Chen, who analyzes protease autoprocessing in HIV and how this can be used in drug discovery [15]. The other is from Margaret Riley and colleagues who offer their interpretation on the rethinking of the composition of a rational antibiotic arsenal for the 21st century [16]. Thomas Keating explains the resistance mechanism of inhibitors of the Helicobacter pylori glutamate racemase in his Special Report [17].

The final six Reviews of this special focus issue discuss a wide range of infectious diseases, including the discovery of compounds to overcome the action of aminoglycoside-modifying enzymes for treating resistant bacteria [18], the modification of depsipeptide natural products to overcome drug resistance [19] and an analysis of work relating to transition-state inhibitors of purine salvage in malaria [20]. Dustin King and Natalie Strynadka discuss the targeting of metallo betalactamase [21], Richard Lee and colleagues analyze the current state of research relating to novel DHPS inhibitors [22], and, finally, Christian Melander and co-workers discuss how two-component systems could be targeted to combat antibiotic resistance [23].

We wish to thank all of the authors and reviewers across both issues for kindly contributing to this two-part special focus. We hope you enjoy the issues!

\section{Financial \& competing interests disclosure}

The author has no relevant affiliations or financial involvement with any organization or entity with a financial interest in or financial conflict with the subject matter or materials discussed in the manuscript. This includes employment, consultancies, honoraria, stock ownership or options, expert testimony, grants or patents received or pending, or royalties. No writing assistance was utilized in the production of this manuscript.

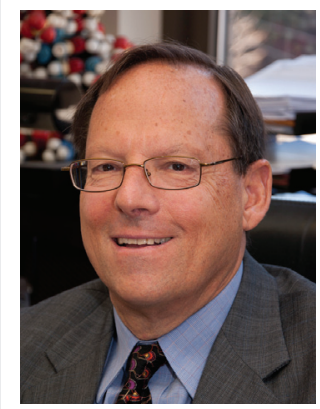

\section{Barry Gold}

Department of Pharmaceutical Sciences, University of Pittsburgh, Pittsburgh, PA 1526I, USA Tel.: + I 4123839593 E-mail: goldbi@pitt.edu 


\section{References}

1 Gold B. Drug resistance: the problem that keeps on giving. Future Med. Chem. 5(9), 981-982 (2013).

2 Ju J, Jiang J, Fesler A. miRNA: the new frontier in cancer medicine. Future Med. Chem. 5(9), 983-985 (2013).

3 Liu R, Wettersten HI, Park S-H, Weiss RH. Small-molecule inhibitors of p21 as novel therapeutics for chemotherapy-resistant kidney cancer. Future Med. Chem. 5(9), 991-994 (2013).

4 Aredia F, Scovassi AI. Manipulation of autophagy in cancer cells: an innovative strategy to fight drug resistance. Future Med. Chem. 5(9), 1009-1021 (2013).

5 Bodle CR, Mackie DI, Roman DL. RGS17: an emerging therapeutic target for lung and prostate cancers. Future Med. Chem. 5(9), 995-1007 (2013).

6 Walton-Diaz A, Khan S, Bourboulia D, Trepel JB, Neckers L, Mollapour M. Contributions of co-chaperones and post-translational modifications towards Hsp90 drug sensitivity. Future Med. Chem. 5(9), 1059-1071 (2013).

7 Cook KL, Clarke PAG, Clarke R. Targeting GRP78 and antiestrogen resistance in breast cancer. Future Med. Chem. 5(9), 1047-1057 (2013).

8 Lecerf-Schmidt F, Peres B, Valdameri G et al. ABCG2: recent discovery of potent and highly selective inhibitors. Future Med. Chem. 5(9), 1037-1045 (2013).
9 Jiang Q, Zheng S, Wang G. Development of new estrogen receptor-targeting therapeutic agents for tamoxifen-resistant breast cancer. Future Med. Chem. 5(9), 1023-1035 (2013).

10 Manchester JI. A fresh start in the hunt for novel antibacterials. Future Med. Chem. 5(11), 1185-1187 (2013).

11 Anderson AC. Possible paths and potential barriers to successfully modeling drug resistance. Future Med. Chem. 5(11), 1181-1183 (2013).

12 Latour JM, Duarte V. PerR: a bacterial resistance regulator and can we target it? Future Med. Chem. 5(11), 1177-1179 (2013).

13 Schiffer C. Interview with Celia Schiffer. Future Med. Chem. 5(11), 1193-1197 (2013).

14 Matthiessen L. Interview with Line Matthiessen. Future Med. Chem. 5(11), 1199-1201 (2013).

15 Huang L, Chen C. Understanding HIV protease autoprocessing for novel therapeutic development. Future Med. Chem. 5(11), 1215-1229 (2013).

16 Riley MA, Robinson SM, Roy CM, Dorit RL. Rethinking the composition of a rational antibiotic arsenal for the $21 \mathrm{st}$ century. Future Med. Chem. 5(11), 1231-1242 (2013).
17 Keating TA. Resistance mechanism to an uncompetitive inhibitor of a single-substrate, single-product enzyme: a study of Helicobacter pylori glutamate racemase. Future Med. Chem. 5(11), 1203-1214 (2013).

18 Labby KJ, Garneau-Tsodikova S. Strategies to overcome the action of AMEs for treating resistant bacterial infections. Future Med. Chem. 5(11), 1285-1309 (2013).

19 Bionda N, Pitteloud J-P, Cudic P. Cyclic lipodepsipeptides: a new class of antibacterial agents in the battle against resistant bacteria Future Med. Chem. 5(11), 1311-1330 (2013).

20 Ducati RG, Namanja-Magliano HA, Schramm VL. Transition-state inhibitors of purine salvage and other prospective enzyme targets in malaria. Future Med. Chem. 5(11), 1341-1360 (2013).

21 King DT, Strynadka NCJ. Targeting metallo$\beta$-lactamase enzymes in antibiotic resistance. Future Med. Chem. 5(11), 1243-1263 (2013).

22 Hammoudeh DI, Zhao Y, White SW, Lee RE. Replacing sulfa drugs with novel DHPS inhibitors. Future Med. Chem. 5(11), 1331-1340 (2013).

23 Worthington RJ, Blackledge MS, Melander C. Small molecule inhibition of bacterial two-component systems to combat antibiotic resistance and virulence. Future Med. Chem. 5(11), 1265-1284 (2013). 